\title{
MMW-HOTLINE
}

Leser der MMW können sich ab sofort mit allen Fragen zur Abrechnung und Praxisführung an Helmut Walbert, Würzburg, wenden. Sie erreichen inn jeden Donnerstag von 13 bis 15 Uhr unter der kostenlosen Rufnummer (0800) 2379830.

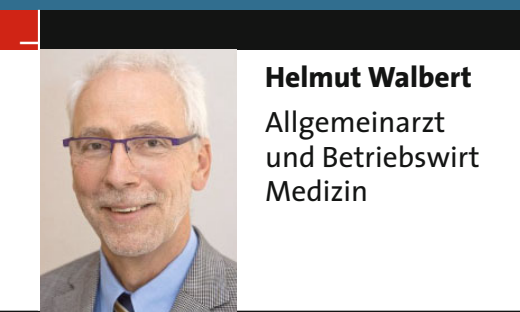

\section{Zahlt die GKV oder ist das eine IGeL?}

Dr. med. G., Allgemeinarzt, Dinslaken: Ich habe eine Jugendliche mit häufigen Allergien, die den Berufswunsch „Friseurin“ hat. Sie möchte vorab getestet werden. Wie gehe ich vor? Kann dies zulasten der Gesetzlichen Krankenversicherung (GKV) abgerechnet werden, oder muss die $\mathrm{Pa}$ tientin die Testung selbst zahlen?

Antwort: Weder noch! Es ist richtig, dass die vorsorgliche Testung keine Leistung der GKV ist. Der Weg der Überweisung sollte auch deshalb unterbleiben, weil dann letztendlich die Leistung zulasten des Facharztbudgets und damit aus der budgetierten Gesamtvergütung vergütet würde.

Die Testung muss auch nicht als Individuelle Gesundheitsleistung laufen. Im Rahmen der Jugendarbeitsschutzuntersuchung können Sie der Patientin als Über-

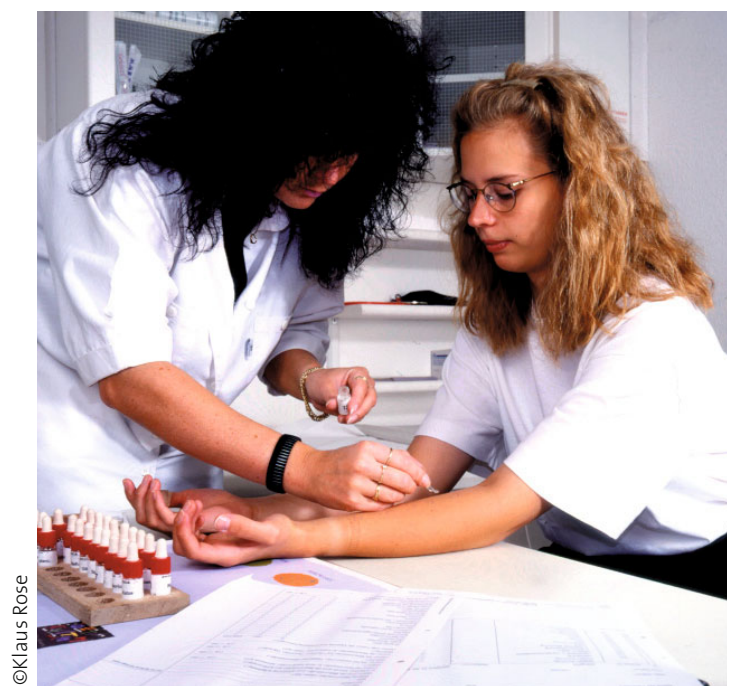

Lieber vorher testen, als die Ausbildung wegen einer Dermatose vorzeitig abbrechen zu müssen. weisung einen speziellen Vordruck im Rahmen des Jugendarbeitsschutzgesetzes ausstellen. Der Dermatologe kann dann die notwendigen Untersuchungen zulasten des entsprechenden Kostenträgers, der Landesbehörde, abrechnen.

Von dieser präventiven Untersuchung sollte vor allem im Rahmen der Jugendarbeitsschutzuntersuchung viel häufiger Gebrauch gemacht werden, um einen unnötigen Abbruch der Ausbildung oder ein frühzeitiges Ende der beruflichen Tätigkeit durch eine Berufsdermatose zu vermeiden.

\section{Konsiliargespräch}

\section{Wie rechne ich das $a b$ ?}

Dr. med. K.H., Allgemeinarzt, München: Welche Abrechnungsmöglichkeiten gibt es für ein Konsiliargespräch mit dem Krankenhausarzt?

Antwort: Im EBM gibt es leider für das Konsiliargespräch mit einem weiter behandelnden Krankenhausarzt keine Gebührenordnungsposition mehr. Die alte Gebührenordnungsposition (GOP) 43 des EBM 96 ist in den Anhang 1 verschwunden. Nur die GOÄ hat noch eine eigene GOP 60, ,Konsiliarische Erörterung zwischen zwei oder mehr liquidationsberechtigten Ärzten, für jeden Arzt“, 120 Punkte, € 6,99 einfach. Da dieses Gespräch in der Regel „dringend und unverzüglich“ mit dem Gesprächspartner ausgeführt wird, kann der Zuschlag GOP E, „Zuschlag für dringend angeforderte und unverzüglich erfolgte Ausführung" zusätzlich abgerechnet werden (16o Punkte oder € 9,33). Der Zuschlag ist nicht steigerungsfähig.

In diesem Zusammenhang sei auf eine meist vergessene Möglichkeit der Kos- tenerstattung hingewiesen: Telefonkostenerstattung im Zusammenhang mit Telefonaten mit dem Krankenhaus zur Vereinbarung einer stationären Aufnahme. Hier müssen in dringenden Fällen sehr oft einige Krankenhäuser angerufen werden, bis man ein Bett für seinen Patienten erhält. Die entstehenden Kosten werden am Tag der Leistungserbringung mit dem Buchstaben „F“ für Ferngespräch und dem entstandenen Betrag in Euro und Cent, also beispielsweise $F € 1,20$, abgerechnet. 